\title{
GALOIS THEORY AND INTEGRAL MODELS OF $\Lambda$-RINGS
}

\author{
JAMES BORGER, BART DE SMIT
}

\begin{abstract}
We show that any $\Lambda$-ring, in the sense of Riemann-Roch theory, which is finite étale over the rational numbers and has an integral model as a $\Lambda$-ring is contained in a product of cyclotomic fields. In fact, we show that the category of them is described in a Galois-theoretic way in terms of the monoid of pro-finite integers under multiplication and the cyclotomic character. We also study the maximality of these integral models and give a more precise, integral version of the result above. These results reveal an interesting relation between $\Lambda$-rings and class field theory.
\end{abstract}

\section{INTRODUCTION}

According to the most common definition, a $\Lambda$-ring structure on a commutative ring $R$ is a sequence of set maps $\lambda_{1}, \lambda_{2}, \ldots$ from $R$ to itself that satisfy certain complex implicitly stated axioms. This notion was introduced by Grothendieck [5], under the name special $\lambda$-ring, to give an abstract setting for studying the structure on Grothendieck groups inherited from exterior power operations; and as far as we are aware, with just one exception [2], $\Lambda$-rings have been studied in the literature for this purpose only.

However, it seems that the study of abstract $\Lambda$-rings - those having no apparent relation to $K$-theory - will have something to say about number theory. One example of such a relationship is the likely existence of strong arithmetic restrictions on the complexity of finitely generated rings that admit a $\Lambda$-ring structure. The primary purpose of this paper is to investigate this issue in the zero-dimensional case. Precisely, what finite étale $\Lambda$-rings over $\mathbf{Q}$ are of the form $\mathbf{Q} \otimes A$, where $A$ is a $\Lambda$-ring that is finite flat over $\mathbf{Z}$ ?

We will consider $\Lambda$-actions only on rings whose underlying abelian group is torsion free, and giving a $\Lambda$-action on such a ring $R$ is the same as giving commuting ring endomorphisms $\psi_{p}: R \rightarrow R$, one for each prime $p$, lifting the Frobenius map modulo $p$-that is, such that $\psi_{p}(x)-x^{p} \in p R$ for all $x \in R$. (The equivalence of this with Grothendieck's original definition is proved in Wilkerson [8].) An example that will be important here is $\mathbf{Z}\left[\mu_{r}\right]=\mathbf{Z}[z] /\left(z^{r}-1\right)$, where $r$ is a positive integer and $\psi_{p}$ sends $z$ to $z^{p}$. A morphism of torsion-free $\Lambda$-rings is the same as a ring map $f$ that satisfies $f \circ \psi_{p}=\psi_{p} \circ f$ for all primes $p$.

Note that if $R$ is a $\mathbf{Q}$-algebra, the congruence conditions in the definition above disappear. Also, Galois theory as interpreted by Grothendieck gives an antiequivalence between the category of finite étale $\mathbf{Q}$-algebras and the category of finite discrete sets equipped with a continuous action of the absolute Galois group $G_{\mathbf{Q}}$ with respect to a fixed algebraic closure $\overline{\mathbf{Q}}$. Combining these two remarks, we

Date: October 22, 2007. 16:12.

Mathematics Subject Classification: 13K05 (primary); 11R37, 19L20, 16W99 (secondary). 
see that the category of $\Lambda$-rings that are finite étale over $\mathbf{Q}$ is nothing more than the category of finite discrete sets equipped with a continuous action of the monoid $G_{\mathbf{Q}} \times \mathbf{N}^{\prime}$, where $\mathbf{N}^{\prime}$ is the monoid $\{1,2, \ldots\}$ under multiplication with the discrete topology. This is because $\mathbf{N}^{\prime}$ is freely generated as a commutative monoid by the prime numbers.

It is not always true, however, that such a $\Lambda$-ring $K$ has an integral $\Lambda$-model, by which we mean a sub- $\Lambda$-ring $A$, finite over $\mathbf{Z}$, such that $\mathbf{Q} \otimes A=K$. In order to formulate exactly when this happens, we write $\hat{\mathbf{Z}}^{\circ}$ for the set of profinite integers viewed as a topological monoid under multiplication, and we consider the continuous monoid map

$$
G_{\mathbf{Q}} \times \mathbf{N}^{\prime} \longrightarrow \hat{\mathbf{Z}}^{\circ}
$$

given by the cyclotomic character $G_{\mathbf{Q}} \rightarrow \hat{\mathbf{Z}}^{*} \subset \hat{\mathbf{Z}}^{\circ}$ on the first factor and the natural inclusion on the second. Note that this map has a dense image. Now let $K$ be a finite étale algebra over $\mathbf{Q}$, and let $S$ be the set of ring maps from $K$ to $\overline{\mathbf{Q}}$. Suppose $K$ is endowed with a $\Lambda$-ring structure, so that we have an induced monoid map

$$
G_{\mathbf{Q}} \times \mathbf{N}^{\prime} \longrightarrow \operatorname{Map}(S, S)
$$

where $\operatorname{Map}(S, S)$ is the monoid of set maps from $S$ to itself. With the discrete topology on $\operatorname{Map}(S, S)$ this map is continuous.

0.1. Theorem. The $\Lambda$-ring $K$ has an integral $\Lambda$-model if and only if the action of $G_{\mathbf{Q}} \times \mathbf{N}^{\prime}$ on $S$ factors (necessarily uniquely) through $\hat{\mathbf{Z}}^{\circ}$; in more precise terms, if and only if there is a continuous monoid map $\hat{\mathbf{Z}}^{\circ} \rightarrow \operatorname{Map}(S, S)$ so that the diagram

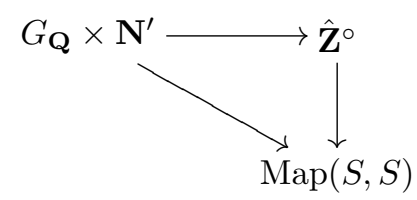

commutes.

It follows that the category of such $\Lambda$-rings is anti-equivalent to the category of finite discrete sets with a continuous action of $\hat{\mathbf{Z}}^{\circ}$ and that every such $\Lambda$-ring is contained in a product of cyclotomic fields. It also suggests there is an interesting theory of a $\Lambda$-algebraic fundamental monoid, analogous to that of the usual algebraic fundamental group, but we will leave this for a later date.

Another consequence is that the elements $-1,0 \in \hat{\mathbf{Z}}^{\circ}$ give an involution $\psi_{-1}$ (complex conjugation) and a idempotent endomorphism $\psi_{0}$ on any $K$ with an integral $\Lambda$-model. In $K$-theory, the dual and rank also give such operators, but here they come automatically from the $\Lambda$-ring structure. Also observe that any element of the subset $0 S \subseteq S$ is Galois invariant and hence corresponds to a direct factor $\mathbf{Q}$ of the algebra $K$. Therefore $K$ cannot be a field unless $K=\mathbf{Q}$.

In the first section, we give some basic facts and also show the sufficiency of the condition in the theorem above. In the second section, we show the necessity. The proof combines a simple application of the Kronecker-Weber theorem and the Chebotarev density theorem with some elementary but slightly intricate work on actions of the monoid $\hat{\mathbf{Z}}^{\circ}$.

The third section gives a proof of the following theorem:

0.2. Theorem. The $\Lambda$-ring $\mathbf{Z}\left[\mu_{r}\right]$ is the maximal integral $\Lambda$-model of $\mathbf{Q} \otimes \mathbf{Z}\left[\mu_{r}\right]$. 
Of course, the non- $\Lambda$ version of this statement is false - the usual maximal order of $\mathbf{Q} \otimes \mathbf{Z}\left[\mu_{r}\right]$ is a product of rings of integers in cyclotomic fields and strictly contains $\mathbf{Z}\left[\mu_{r}\right]$, if $r>1$.

A direct consequence of these theorems is:

0.3. Corollary. Every $\Lambda$-ring that has finite rank as an abelian group and has no non-zero nilpotent elements is a sub- $\Lambda$-ring of a $\Lambda$-ring of the form $\mathbf{Z}\left[\mu_{r}\right]^{n}$.

We do not need to require that the ring be torsion free because any torsion element in a $\Lambda$-ring is nilpotent, by an easy lemma attributed to G. Segal [3, p. 295]. We emphasize that while the definition of $\Lambda$-ring that we gave above does not literally require the ring to be torsion free, it is not the correct definition in the absence of this assumption. In particular, Segal's lemma and the corollary above are false if the naive definition is used; for example, take a finite field. For the definition of $\Lambda$-ring in the general case, see [5], [8], or [1].

Finally, many of the questions answered in this paper have analogues over general number fields. There one would use Frobenius lifts modulo prime ideals of the ring of integers, and then general class field theory and the class group come in, as well as the theory of complex multiplication in particular cases. Because these analogues of $\Lambda$-rings are not objects of prior interest, we have not included anything about them here. But it is clear that finite-rank $\Lambda$-rings, in this generalized sense or the original, are fundamentally objects of class field theory and that they offer a slightly different perspective on the subject. It would be interesting to explore this further.

\section{BASICS}

The category of $\Lambda$-rings has all limits and colimits, and they agree, as rings, with those taken in the category of rings. (E.g. [1]) We will only need to take tensor products, intersections, and images of morphisms, and it is quite easy to show their existence on the subcategory of torsion-free $\Lambda$-rings using the equivalent definition given in the introduction. For any ring $R$, let $R\left[\mu_{r}\right]$ denote $R[z] /\left(z^{r}-1\right)$. Because $R\left[\mu_{r}\right]=R \otimes \mathbf{Z}[z] /\left(z^{r}-1\right)$, the ring $R\left[\mu_{r}\right]$ is naturally a $\Lambda$-ring if $R$ is.

Given a $\Lambda$-ring $R$, it will be convenient to call a $\Lambda$-ring $K$ equipped with a map $R \rightarrow K$ of $\Lambda$-rings an $R \Lambda$-ring. (Compare [1, 1.13].) When we say $K$ is flat, or étale, or so on, we mean as $R$-algebras in the usual sense. We call a sub- $\Lambda$-ring of a $\mathbf{Q} \Lambda$-ring a $\Lambda$-order if it is finite over $\mathbf{Z}$. We do not require that it have full rank.

1.1. Proposition. Let $K$ be a finite étale $\mathbf{Q} \Lambda$-ring. Then $K$ has a $\Lambda$-order that contains all others.

We call this $\Lambda$-order the maximal $\Lambda$-order of $K$.

Proof. Because any $\Lambda$-order $A$ is contained in the usual maximal order of $K$, which is finite over $\mathbf{Z}$, it is enough to show that any two $\Lambda$-orders $A$ and $B$ are contained in a third. But $A \otimes B$ is a $\Lambda$-ring that is finite over $\mathbf{Z}$. Since $A \otimes B$ is the coproduct in the category of $\Lambda$-rings, the map $A \otimes B \rightarrow K$ coming from the universal property of coproducts (i.e., $a \otimes b \mapsto a b$ ) is a $\Lambda$-ring map. Therefore its image is a $\Lambda$-ring that is finite over $\mathbf{Z}$, is contained in $K$, and contains $A$ and $B$.

1.2. Proposition. Let $K \subseteq L$ be an inclusion of finite étale $\mathbf{Q} \Lambda$-rings. Let $A \subseteq K$ and $B \subseteq L$ be their maximal $\Lambda$-orders. Then $A=K \cap B$. 
Proof. The intersection $K \cap B$ is on the one hand a sub- $\Lambda$-ring of $K$ and, on the other, finite over $\mathbf{Z}$. It is maximal among such rings because of the maximality of $B$.

We can now prove the sufficiency of the conditions of theorem 0.1 . For any ring $R$, let $R^{\circ}$ denote $R$ itself but viewed only as a monoid under multiplication. So the group $R^{*}$ of units is just the group of invertible elements of the monoid $R^{\circ}$.

1.3. Proposition. Let $r$ be a positive integer, let $S$ be a finite $(\mathbf{Z} / r \mathbf{Z})^{\circ}$-set, and let $K$ be the corresponding finite étale $\mathbf{Q} \Lambda$-ring. Then $K$ has an integral $\Lambda$-model.

Proof. Take a set $T$ (such as $S$ ) admitting a surjection $\coprod_{T}(\mathbf{Z} / r \mathbf{Z})^{\circ} \rightarrow S$ of $(\mathbf{Z} / r \mathbf{Z})^{\circ}$ sets, the left side denoting the free $(\mathbf{Z} / r \mathbf{Z})^{\circ}$-set generated by $T$. Let $L$ be the corresponding finite étale $\mathbf{Q} \Lambda$-ring. Then $K$ is naturally a sub- $\Lambda$-ring of $L$. On the other hand $L$ is $\mathbf{Q}\left[\mu_{r}\right]^{T}$ and so has a $\Lambda$-model $\mathbf{Z}\left[\mu_{r}\right]^{T}$. The intersection of this with $K$ is then both a $\Lambda$-ring and an order of full rank in $K$.

\section{NECESSARY CONDITIONS}

Let $K$ be a finite étale $\mathbf{Q} \Lambda$-ring admitting an integral $\Lambda$-model $A$, and let $S=$ $\operatorname{Hom}(K, \overline{\mathbf{Q}})$ be the corresponding $G_{\mathbf{Q}} \times \mathbf{N}^{\prime}$-set.

The purpose of this section is to show that there is an integer $r>0$ such that this action factors through the map $G_{\mathbf{Q}} \times \mathbf{N}^{\prime} \rightarrow(\mathbf{Z} / r \mathbf{Z})^{\circ}$ given by the cyclotomic character on the first factor and reduction modulo $r$ on the second.

As usual, we say a prime number $p$ is unramified in $A$ if $A / p A$ has no non-zero nilpotent elements. A prime is ramified in $A$ if and only if it divides the discriminant of $A$. Therefore the set of primes that ramify in $A$ is finite and contains the set of primes that ramify in the usual maximal order of $K$.

2.1. Proposition. The endomorphism $\psi_{p}$ of $A$ is an automorphism if and only if $p$ is unramified in $A$. In this case, $\psi_{p}$ is the unique lift of the Frobenius endomorphism of $A / p A$.

Proof. If $\psi_{p}$ is an automorphism, then the Frobenius endomorphism $x \mapsto x^{p}$ of $A / p A$ is an automorphism, and so $p$ is unramified.

Suppose instead that $p$ is unramified. Then $A / p A$ is a finite product of finite fields, and so the Frobenius endomorphism of $A / p A$ is an automorphism of finite order. The category of finite étale $\mathbf{Z}_{p}$-algebras is equivalent to the category of finite étale $\mathbf{F}_{p}$-algebras, by way of the functor $\mathbf{F}_{p} \otimes_{\mathbf{z}_{p}}-$. (See [4, IV (18.3.3)], say). Thus the endomorphism $1 \otimes \psi_{p}$ of $\mathbf{Z}_{p} \otimes A$ is the unique Frobenius lift and it is an automorphism of finite order. It follows that $\psi_{p}: A \rightarrow A$ is the unique Frobenius lift to $A$, and is also an automorphism.

2.2. Proposition. There is a positive integer $c$, divisible only by primes that ramify in $A$, such that the action of $G_{\mathbf{Q}}$ on $S$ factors through the cyclotomic character $G_{\mathbf{Q}} \rightarrow(\mathbf{Z} / c \mathbf{Z})^{*}$. If $p$ is unramified in $A$, then $p \in \mathbf{N}^{\prime}$ and $(p \bmod c) \in(\mathbf{Z} / c \mathbf{Z})^{*}$ act in the same way on $S$.

Proof. Define the number field $N$ to be the invariant field of the kernel of the map $G_{\mathbf{Q}} \rightarrow \operatorname{Map}(S, S)$. Write $\bar{G}=\operatorname{Gal}(N / \mathbf{Q})$ and let $\mathcal{O}_{N}$ be the ring of integers of $N$.

Take any element $g \in \bar{G}$. By Chebotarev's theorem [7, V.6] there is an unramified prime $\mathfrak{p}$ of $N$ lying over a prime number $p$ such that $g(x) \equiv x^{p} \bmod \mathfrak{p}$ for all $x \in \mathcal{O}_{N}$, i.e., $g$ is the Frobenius element of $\mathfrak{p}$ in the extension $\mathbf{Q} \subset N$. Since Chebotarev's 
theorem provides infinitely many such $\mathfrak{p}$, we may also assume that $A$ is unramified at $p$.

We now claim that for all $s \in S=\operatorname{Hom}\left(A, \mathcal{O}_{N}\right)$ the maps $s \circ \psi_{p}$ and $g \circ s$ from $A$ to $\mathcal{O}_{N}$ are equal. Since $A$ is unramified at $p$, the map $\operatorname{Hom}\left(A, \mathcal{O}_{N}\right) \rightarrow \operatorname{Hom}\left(A, \mathcal{O}_{N} / \mathfrak{p}\right)$ is injective, so it suffices to show that their compositions with the map $\mathcal{O}_{N} \rightarrow \mathcal{O}_{N} / \mathfrak{p}$ are equal. But this follows from 2.1 and our choice of $\mathfrak{p}$. Thus, $g \in \bar{G}$ and $p \in \mathbf{N}^{\prime}$ act in the same way on $S$.

It follows that the image of $G_{\mathbf{Q}}$ in $\operatorname{Map}(S, S)$ is contained in the image of $\mathbf{N}^{\prime}$, so $\bar{G}$ is abelian. By the Kronecker-Weber theorem [7, III.3.8], $N$ is contained in a cyclotomic field $\mathbf{Q}\left(\mu_{c}\right)$, where $c$ is divisible only by primes that ramify in $N$. Since $N$ is the common Galois closure of the components of $A \otimes \mathbf{Q}$, such primes are ramified in $A$ as well. The last statement follows from the fact that for any prime number $p \nmid c$ the element $(p \bmod c) \in(\mathbf{Z} / c \mathbf{Z})^{*}$ corresponds to the Frobenius element of any prime over $p$ in the extension $\mathbf{Q} \subset \mathbf{Q}\left(\mu_{c}\right)$.

It follows that our map of topological monoids $G_{\mathbf{Q}} \times \mathbf{N}^{\prime} \rightarrow \operatorname{Map}(S, S)$ factors through $\hat{\mathbf{Z}}^{*} \times \mathbf{N}^{\prime}$. We will show that it factors further through $\hat{\mathbf{Z}}^{\circ}$ with the following criterion.

2.3. Proposition. A continuous action of $\hat{\mathbf{Z}}^{*} \times \mathbf{N}^{\prime}$ on a finite discrete set $T$ factors through a continuous action of $\hat{\mathbf{Z}}^{\circ}$ if and only if

(i) all but finitely many primes $p \in \mathbf{N}^{\prime}$ act as automorphisms on $T$, and

(ii) for all $d \in \mathbf{N}^{\prime}$ there exists an integer $c_{d}$ such that the action of $\hat{\mathbf{Z}}^{*}$ on $d T$ factors through $\left(\mathbf{Z} / c_{d} \mathbf{Z}\right)^{*}$ and for each $n \in \mathbf{N}^{\prime}$ with $n d T=d T$ we have

- $n$ is relatively prime to $c_{d}$, and

- the elements $\left(n \bmod c_{d}\right) \in\left(\mathbf{Z} / c_{d} \mathbf{Z}\right)^{*}$ and $n \in \mathbf{N}^{\prime}$ act on $d T$ in the same way.

Proof. To show the necessity of (i) and (ii), assume that the action of $\hat{\mathbf{Z}}^{*} \times \mathbf{N}^{\prime}$ factors through $(\mathbf{Z} / r \mathbf{Z})^{\circ}$ for some integer $r>0$. Then all primes not dividing $r$, when viewed as elements of $\mathbf{N}^{\prime}$, act as automorphisms on $T$. This establishes (i). To show (ii), take any $d \in \mathbf{N}^{\prime}$ and let $c_{d}$ be the smallest positive integer $c$ for which the $\hat{\mathbf{Z}}^{*}$-action on $d T$ factors through $(\mathbf{Z} / c \mathbf{Z})^{*}$. Note that $c_{d}$ divides all $c$ with this property. Now suppose that $p$ is a prime with $p d T=d T$. Write $r=p^{n} e$, with $p \nmid e$. Then for any $x, y \in(\mathbf{Z} / r \mathbf{Z})^{\circ}$ with $x \equiv y \bmod e$ and any $s \in d T$ (in fact any $s \in T$ ), we have

$$
p^{n}(x s)=\left(p^{n} x\right) s=\left(p^{n} y\right) s=p^{n}(y s) .
$$

Since $p$ acts bijectively on $d T$, this implies that $x$ and $y$ act in the same way on $d T$, so the action of $\hat{\mathbf{Z}}^{\circ}$ on $d T$ factors through $(\mathbf{Z} / e \mathbf{Z})^{\circ}$. In particular, $c_{d} \mid e$, so $p \nmid c_{d}$, and the elements $p \in \mathbf{N}^{\prime}$ and $(p \bmod e) \in(\mathbf{Z} / e \mathbf{Z})^{*}$ and $\left(p \bmod c_{d}\right) \in\left(\mathbf{Z} / c_{d} \mathbf{Z}\right)^{*}$ all act in the same way on $d T$. Since $\mathbf{N}^{\prime}$ is generated by the primes, part (ii) follows.

For the converse, suppose (i) and (ii) hold. For every prime number $p$, let $a_{p}$ be the smallest integer $a \geq 0$ such that $p^{a} T=p^{a+1} T$. By (i) we have $a_{p}=0$ for all but finitely many $p$, so $r_{0}=\prod_{p} p^{a_{p}}$ is an integer. Note that for any $n \in \mathbf{N}^{\prime}$ we have $n T=\operatorname{gcd}\left(n, r_{0}\right) T$.

Now let $r$ be any integer divisible by $d c_{d}$ for every $d \mid r_{0}$. We will show that the action of $\hat{\mathbf{Z}}^{*} \times \mathbf{N}^{\prime}$ on $T$ factors through $(\mathbf{Z} / r \mathbf{Z})^{\circ}$. To do this, we will show directly that any two elements $\left(a_{1}, d_{1}\right),\left(a_{2}, d_{2}\right) \in \hat{\mathbf{Z}}^{*} \times \mathbf{N}^{\prime}$ satisfying $a_{1} d_{1} \equiv a_{2} d_{2} \bmod r$ act in the same way on $T$. 
Since $r_{0} \mid r$ the congruence implies that $d_{1}$ and $d_{2}$ have the same greatest common divisor $d$ with $r_{0}$, so we have $d_{1} T=d T=d_{2} T$. For $i=1,2$ we define $d_{i}^{\prime} \in \mathbf{N}^{\prime}$ by $d_{i}=d d_{i}^{\prime}$ and deduce that $d_{i}^{\prime}(d T)=d T$. Using (ii) one sees that $d_{i}^{\prime}$ is coprime to $c_{d}$, and that the action of $d_{i}^{\prime}$ on $d T$ is that of $\left(d_{i}^{\prime} \bmod c_{d}\right) \in\left(\mathbf{Z} / c_{d} \mathbf{Z}\right)^{*}$. By the defining property of $r$ we have $d c_{d} \mid r$, so $a_{1} d_{1}^{\prime} d \equiv a_{2} d_{2}^{\prime} d \bmod d c_{d}$, which implies $a_{1} d_{1}^{\prime} \equiv a_{2} d_{2}^{\prime} \bmod c_{d}$. It follows that $\left(a_{1}, d_{1}^{\prime}\right)$ and $\left(a_{2}, d_{2}^{\prime}\right)$ are mapped to the same element of $\left(\mathbf{Z} / c_{d} \mathbf{Z}\right)^{\circ}$, which in fact lies in $\left(\mathbf{Z} / c_{d} \mathbf{Z}\right)^{*}$. Thus, $\left(a_{1}, d_{1}^{\prime}\right)$ and $\left(a_{2}, d_{2}^{\prime}\right)$ act identically on $d T$, and composing with $(1, d)$ we see that $\left(a_{1}, d_{1}\right)$ and $\left(a_{2}, d_{2}\right)$ act in the same way on $T$.

In order to finish the proof of theorem 0.1 one checks the conditions of 2.3 for $T=S$. Condition (i) follows from 2.1 and the fact that $A$ is ramified at only finitely many primes. For condition (ii), suppose $d \in \mathbf{N}^{\prime}$ is given and consider the sub- $\Lambda$ ring $\psi_{d}(A)$ of $A$ which corresponds to the $G_{\mathbf{Q}} \times \mathbf{N}^{\prime}$-set $d S$ of $S$. Proposition 2.2 applied to $\psi_{d}(A)$ now provides an integer $c_{d}$ so that the $G_{\mathbf{Q}^{-a c t i o n}}$ on $d S$ factors through $\left(\mathbf{Z} / c_{d} \mathbf{Z}\right)^{*}$. Any $n \in \mathbf{N}^{\prime}$ with $n d S=d S$ is a product of primes that are unramified in $\psi_{d}(A)$ by 2.1 , and so 2.2 tells us that $\left(n \bmod c_{d}\right) \in\left(\mathbf{Z} / c_{d} \mathbf{Z}\right)^{*}$ and that this element acts on $d S$ in the same way as $n$. This gives condition (ii).

\section{Explicit maximal $\Lambda$-ORDERS}

Given a prime number $p$, there is a notion of $\Lambda_{p}$-action on a ring $R$, and as before, this has a simple description if $R$ has no $p$-torsion: a ring endomorphism $\psi_{p}$ of $R$ that lifts the Frobenius endomorphism, that is, such that $\psi_{p}(x)-x^{p} \in p R$ for all $x \in R$. Also as before, a sub- $\Lambda_{p}$-ring $A$ of a $\mathbf{Q}_{p} \Lambda_{p}$-ring $K$ is called a $\Lambda_{p}$-order if it is finite over $\mathbf{Z}_{p}$. It is said to be maximal if it contains every other $\Lambda_{p}$-order in $K$.

We have two natural ways of making $\Lambda_{p}$-orders. First, for any abelian group $V$ the group ring $\mathbf{Z}_{p}[V]$ is a $\Lambda_{p}$-ring when we set $\psi_{p}(r)=r$ for $r \in \mathbf{Z}_{p}$ and $\psi_{p}(v)=v^{p}$ for $v \in V$. Secondly, if $A$ is the ring of integers of a finite unramified extension $K$ of $\mathbf{Q}_{p}$, then $A$ has a unique $\Lambda_{p}$-ring structure, where $\psi_{p}$ is the Frobenius map (cf. 2.1). By extending $\psi_{p}$ to $K$ we see that $A$ is the maximal $\Lambda_{p}$-order of $K$. Taking tensor products of these two building blocks we see that for any integer $q$ the ring $A\left[\mu_{q}\right]=A[z] /\left(z^{q}-1\right)$ is a $\Lambda_{p}$-order in $K\left[\mu_{q}\right]$.

3.1. Lemma. If $q$ is a power of $p$, then $A\left[\mu_{q}\right]$ is the maximal $\Lambda_{p}$-order of $K\left[\mu_{q}\right]$.

Proof. By induction, it is enough to assume $A\left[\mu_{q}\right]$ is maximal and then prove $A\left[\mu_{p q}\right]$ is.

Let $k$ denote the residue field of $A$, and let $\zeta$ denote a primitive $p q$-th root of unity in some extension of $K$. Then we have $K[z] /\left(z^{p q}-1\right)=K(\zeta) \times K[y] /\left(y^{q}-\right.$ $1)$, the element $z$ corresponding to $(\zeta, y)$. In these terms, the $\Lambda_{p}$-action is given by $\psi_{p}(b, f(y))=\left(f^{*}\left(\zeta^{p}\right), f^{*}\left(y^{p}\right)\right)$, where $f^{*}(y)$ denotes polynomial obtained by applying the Frobenius map $\psi_{p}$ coefficient-wise to $f(y)$.

Now consider the following diagram of rings:

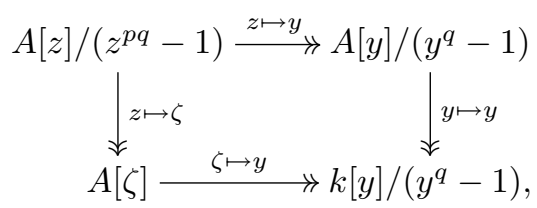


where $A[\zeta]$ denotes the ring of integers in $K(\zeta)$. As noted for example in KervaireMurthy [6], this is a pull-back diagram. This is just an instance of the easy fact that for ideals $I$ and $J$ in any ring $R$, we have

$$
R /(I \cap J)=R / I \times_{R /(I+J)} R / J .
$$

In our case, take $R=A[z], I=\left(z^{q}-1\right)$, and $J=\left(1+z^{q}+\cdots+z^{q(p-1)}\right)$.

Now suppose $R$ is a $\Lambda_{p}$-order in $K\left[\mu_{p q}\right]$. Then the image of $R$ in $K[y] /\left(y^{q}-1\right)$ is contained, by induction, in $A[y] /\left(y^{q}-1\right)$. Therefore $R$ is contained in $A[\zeta] \times$ $A[y] /\left(y^{q}-1\right)$; we will view elements of $R$ as elements of this product without further comment. Because the diagram above is a pull-back diagram, we need only show that the two maps $R \rightrightarrows k[y] /\left(y^{q}-1\right)$ given by mapping to the two factors and then projecting to $k[y] /\left(y^{q}-1\right)$ agree.

Let $v$ denote the valuation on $A[\zeta]$ normalized so that $v(p)=1$. Let $a \leqslant 1 /(p-1)$ be the largest number such that

$$
\text { for all }(b, f(y)) \in R \text { we have } v(b-f(\zeta)) \geqslant a \text {. }
$$

Note that the expression $f(\zeta)$ makes sense only modulo $\zeta_{p}-1$, and so the condition above is meaningless if $a>v\left(\zeta_{p}-1\right)=1 /(p-1)$.

Let $(b, f(y))$ be an element of $R$. Then because $R$ is a $\Lambda_{p}$-ring, there is an element $(c, g(y)) \in p R$ such that

$$
(c, g(y))=(b, f(y))^{p}-\psi_{p}(b, f(y))=\left(b^{p}-f^{*}\left(\zeta^{p}\right), f(y)^{p}-f^{*}\left(y^{p}\right)\right) .
$$

On the other hand, because of our assumption on $a$, we have

$$
1+a \leqslant v(c-g(\zeta))=v\left(\left(b^{p}-f^{*}\left(\zeta^{p}\right)\right)-\left(f(\zeta)^{p}-f^{*}\left(\zeta^{p}\right)\right)=v\left(b^{p}-f(\zeta)^{p}\right) .\right.
$$

But because the integral polynomial $p(X-Y)$ divides $(X-Y)^{p}-\left(X^{p}-Y^{p}\right)$, we have

$$
v\left((b-f(\zeta))^{p}-\left(b^{p}-f(\zeta)^{p}\right)\right) \geqslant 1+a
$$

and hence $v(b-f(\zeta)) \geqslant(1+a) / p$. That is, $(1+a) / p$ satisfies (3.1.1).

But then $a=1 /(p-1)$ because otherwise this would violate the maximality of $a$. In other words, for any element $(b, f(y)) \in R$, the element $\zeta_{p}-1$ divides $b-f(\zeta)$. This is just another way of saying $b$ and $f(y)$ have the same image in $A[\zeta] /\left(\zeta_{p}-1\right)=k[y] /\left(y^{q}-1\right)$, and hence the element $(b, f(y))$ lies in the fiber product, which we showed above is $A\left[\mu_{p q}\right]$.

3.2. Remark. It is not true that $\mathbf{Z}_{p}[V]$ is maximal for every finite abelian group $V$. For example, if $V=\mathbf{Z} / p \mathbf{Z} \times \mathbf{Z} / p \mathbf{Z}$ and $x=\frac{1}{p} \sum_{\sigma \in V} \sigma$, then $\psi_{p}(x)=p$ and $x^{2}=p x$. Therefore, the subring $\mathbf{Z}_{p}[V][x]$ of $\mathbf{Q}_{p}[V]$ is a sub- $\Lambda_{p}$-ring which is finite over $\mathbf{Z}_{p}$. The global analogue also holds: $\mathbf{Z}[V]$ is not the maximal integral $\Lambda$-model for $\mathbf{Q}[V]$. This follows from the local statement.

3.3. Proposition. Let $K$ be a finite étale $\mathbf{Q} \Lambda$-ring, and let $R$ be a $\Lambda$-order. If $\mathbf{Z}_{p} \otimes R$ is a maximal $\Lambda_{p}$-order in $\mathbf{Q}_{p} \otimes R$ for all primes $p$, then $R$ is maximal.

Proof. Let $S$ be the maximal $\Lambda$-order in $\mathbf{Q} \otimes R$. Because $\mathbf{Z}_{p} \otimes R$ is the maximal $\Lambda_{p}$-order in $\mathbf{Q}_{p} \otimes R$, the inclusion $\mathbf{Z}_{p} \otimes R \subseteq \mathbf{Z}_{p} \otimes S$ is an equality. Therefore $R$ and $S$ have the same rank, and $p$ does not divide the index of $R$ in $S$.

3.4. Theorem. Let $r \geqslant 1$ be an integer. Then $\mathbf{Z}\left[\mu_{r}\right]$ is the maximal $\Lambda$-order in $\mathbf{Q}\left[\mu_{r}\right]$. 
Proof. By 3.3, it suffices to show that for every prime $p$, the $\Lambda_{p}$-order $\mathbf{Z}_{p}\left[\mu_{r}\right]$ maximal. Write $r=q n$, where $q$ is the largest power of $p$ dividing $r$. Then $\mathbf{Z}_{p}\left[\mu_{r}\right]=\mathbf{Z}_{p}\left[\mu_{n}\right]\left[\mu_{q}\right]=\prod_{A} A\left[\mu_{q}\right]$, where $A$ runs over the irreducible factors of $\mathbf{Z}_{p}\left[\mu_{n}\right]$, all of which are unramified extensions of $\mathbf{Z}_{p}$. By 3.1, each factor $A\left[\mu_{q}\right]$ is a maximal $\Lambda_{p}$-order, and therefore so is their product.

3.5. Remark. Using 0.3 and 1.2 , we can also describe the maximal $\Lambda$-order in general as follows. Let $S$ be a finite $(\mathbf{Z} / r \mathbf{Z})^{\circ}$-set, let $\zeta_{r} \in \overline{\mathbf{Q}}$ denote a primitive $r$-th root of unity, and let $K=\operatorname{Hom}_{(\mathbf{Z} / r \mathbf{Z})^{*}}\left(S, \mathbf{Q}\left(\zeta_{r}\right)\right)$ denote the corresponding finite étale $\Lambda$-ring over $\mathbf{Q}$. Consider the isomorphism

$$
\mathbf{Q}\left[\mu_{r}\right] \rightarrow \prod_{d \mid r} \mathbf{Q}\left(\zeta_{r}^{d}\right)
$$

given by $z \mapsto\left(\ldots, \zeta_{r}^{d}, \ldots\right)_{d \mid r}$. Then the maximal $\Lambda$-order in $K$ is the set of elements $f \in K$ such that for all $s \in S$, the element

$$
(\ldots, f(d s), \ldots)_{d \mid r} \in \prod_{d \mid r} \mathbf{Q}\left(\zeta_{r}^{d}\right) \cong \mathbf{Q}\left[\mu_{r}\right]
$$

lies in $\mathbf{Z}\left[\mu_{r}\right]$.

\section{REFERENCES}

[1] James Borger and Ben Wieland. Plethystic algebra. Adv. Math., 194(2):246-283, 2005.

[2] F. J. B. J. Clauwens. Commuting polynomials and $\lambda$-ring structures on Z $[x]$. J. Pure Appl. Algebra, 95(3):261-269, 1994.

[3] Andreas W. M. Dress. Induction and structure theorems for orthogonal representations of finite groups. Ann. of Math. (2), 102(2):291-325, 1975.

[4] A. Grothendieck. Éléments de géométrie algébrique. IV. Étude locale des schémas et des morphismes de schémas IV. Inst. Hautes Études Sci. Publ. Math., (32):361, 1967.

[5] Alexander Grothendieck. La théorie des classes de Chern. Bull. Soc. Math. France, 86:137-154, 1958.

[6] Michel A. Kervaire and M. Pavaman Murthy. On the projective class group of cyclic groups of prime power order. Comment. Math. Helv., 52(3):415-452, 1977.

[7] Jürgen Neukirch. Class field theory, volume 280 of Grundlehren der Mathematischen Wissenschaften. Springer-Verlag, Berlin, 1986.

[8] Clarence Wilkerson. Lambda-rings, binomial domains, and vector bundles over $\mathbf{C} P(\infty)$. Comm. Algebra, 10(3):311-328, 1982.

James Borger, Mathematical Sciences Institute, Building 27, Australian National University, ACT 0200, Australia

Bart de Smit, Mathematisch Institudt, Universiteit Leiden, Postbus 9512, 2300 RA Leiden, The Netherlands

E-mail address: borger@maths.anu.edu.au, desmit@math.leidenuniv.nl 